\title{
USO DA RMN COMO UM SENSOR ONLINE EM PROCESSOS INDUSTRIAIS
}

\author{
Fabiana Diuk de Andrade \\ Instituto de Química de São Carlos, Universidade de São Paulo, CP 780, 13560-970 São Carlos - SP, Brasil \\ Luiz Alberto Colnago* \\ Embrapa Instrumentação, Rua XV de Novembro, 1452, 13560-970 São Carlos - SP, Brasil
}

Recebido em 7/12/11; aceito em 24/5/12; publicado na web em 31/8/12

\begin{abstract}
USE OF NMR AS AN ONLINE SENSOR IN INDUSTRIAL PROCESSES. Nuclear magnetic resonance (NMR) is one of the most versatile analytical techniques for chemical, biochemical and medical applications. Despite this great success, NMR is seldom used as a tool in industrial applications. The first application of NMR in flowing samples was published in 1951. However, only in the last ten years Flow NMR has gained momentum and new and potential applications have been proposed. In this review we present the historical evolution of flow or online NMR spectroscopy and imaging, and current developments for use in the automation of industrial processes.
\end{abstract}

Keywords: time-domain NMR; online analysis; industrial processes.

\section{INTRODUÇÃO}

A ressonância magnética nuclear (RMN) é uma das principais ferramentas analíticas utilizadas nas áreas da Física, Química, Biologia, Bioquímica, Agricultura, Engenharia, Ciências dos Materiais e Medicina. ${ }^{1-4}$ Os métodos analíticos tradicionais em via úmida demandam tempo, trabalho e grande quantidade de solventes, além de destruírem a amostra. ${ }^{5}$ Assim, métodos espectroscópicos como a espectroscopia na região do infravermelho próximo (NIR) e RMN, que são rápidos e não destrutivos, vêm sendo cada vez mais utilizados em análises químicas estáticas e online em processos industriais. ${ }^{6} \mathrm{O}$ NIR já vem sendo usado rotineiramente em alguns processos industriais online. ${ }^{7}$ Porém, a RMN tem sido pouco utilizada no controle e certificação de qualidade em processos industriais, devido principalmente aos altos custos dos equipamentos, à necessidade de pessoal especializado e aos equipamentos serem muito sensíveis para uso em ambiente industrial. Essas limitações são válidas para protocolos de RMN em alta resolução em alto campo. ${ }^{8}$

No entanto, muitas dessas limitações não são observadas ou são minimizadas em grande parte das aplicações envolvendo a RMN no domínio do tempo (RMN-DT). Os espectrômetros de RMN-DT normalmente utilizam ímãs permanentes de baixo campo magnético, abaixo de 0,5 T (20 MHz para ${ }^{1} \mathrm{H}$ ), que são aparelhos de pequeno porte, muitas vezes de bancada, de baixo custo, fácil operação e que já estão incorporados a alguns processos industriais no modo offline. ${ }^{9}$

No modo offline, as amostras dos produtos a serem analisados são retiradas da linha de produção e enviadas ao laboratório de controle de qualidade. ${ }^{1}$ Além dessas aplicações offline, a RMN em geral e, principalmente, a RMN-DT tem grande potencial para ser incorporada aos processos produtivos no modo online. Nesse modo as amostras (ou todos os produtos) são analisadas diretamente na linha de produção, com as amostras sendo levadas ao sensor de RMN por tubulações (líquidos) ou por esteiras (sólidos). ${ }^{10}$

Assim, a RMN online vem sendo avaliada como um sensor industrial capaz de analisar, selecionar ou classificar não destrutivamente milhares de amostra por hora, diretamente na linha de processos. ${ }^{10}$ Portanto, apresenta-se aqui uma revisão dos principais artigos de RMN em fluxo (online) tanto com espectrômetros em onda contínua,

*e-mail: colnago@cnpdia.embrapa.br quanto pulsada (domínio do tempo, com transformada de Fourier e imagens) apresentando os produtos e as principais metodologias que estão sendo estudadas.

\section{ANÁLISES EM FLUXO POR RMN EM ONDA CONTÍNUA}

Assim como em outras aplicações da RMN, os primeiros experimentos em fluxo também utilizaram espectrômetros de onda contínua. Nesses espectrômetros é aplicada continuamente uma radiofrequência (rf) na sonda de RMN para gerar um campo magnético oscilante, denominado $\mathrm{B}_{1}$, para excitar as transições dos spins nucleares. Para a observação dos sinais de RMN normalmente faz-se a varredura do campo magnético externo, denominado $\mathrm{B}_{0}$, de modo a satisfazer a condição de ressonância (Equação 1), onde $v$ é frequência de ressonância magnética e $\gamma$ a razão magnetogírica.

$$
v=\frac{\gamma B_{0}}{2 \pi}
$$

A primeira análise em fluxo por RMN foi feita por Suryan em 1951, poucos anos após a descoberta da RMN em 1946. ${ }^{11}$ Nesse trabalho foi utilizado um espectrômetro de RMN em onda contínua baseado em um eletromagneto de $25 \mathrm{MHz}$ para ${ }^{1} \mathrm{H}$. Os experimentos foram baseados na análise dos tempos de relaxação da água em fluxo sob a força da gravidade, passando pela sonda de RMN por meio de um tubo. ${ }^{11}$

Suryan observou dois efeitos significativos no sinal de RMN: um aumento temporário na amplitude do sinal de até 20 vezes (em comparação com o sinal da amostra estática) com um subsequente decréscimo. Observou também que a razão do sinal do líquido em fluxo constante e estático era aproximadamente 5, com dependência da velocidade do fluxo. Concluiu que a magnitude desses efeitos estava condicionada a vários fatores, como o tempo de relaxação longitudinal $\left(\mathrm{T}_{1}\right)$ do líquido, o tempo em que a amostra fica no campo magnético, tanto fora quanto no interior da bobina de rf e a velocidade do fluxo. ${ }^{11}$

Em 1962, Ladner e Stacey utilizaram a RMN em fluxo contínuo para analisar a umidade de carvão. ${ }^{12} \mathrm{O}$ fluxo, sob a força da gravidade, foi controlado a $100 \mathrm{~g}$ por hora. Com os espectros de hidrogênio obtidos com um aparelho de onda contínua de $16 \mathrm{MHz}$ para ${ }^{1} \mathrm{H}$, conseguiram distinguir entre os sinais derivados dos hidrogênios do 
material sólido (carvão) e dos hidrogênios presentes na água associada a esse material. Mediram a variação de umidade de 0 a $14 \%$ com precisão de $\pm 1 \%$. Essa pode ser considerada a primeira aplicação da RMN em fluxo para controle de um processo industrial. ${ }^{12}$

Após essas aplicações iniciais, outros trabalhos foram aperfeiçoando a instrumentação e as metodologias utilizadas para medidas em fluxo. Um desses exemplos pode ser visto no trabalho de McIvor (1969) que utilizou um aparelho comercial de RMN em onda contínua, Perkin Elmer modelo R10, com campo magnético $\mathrm{B}_{0}$ de 1,4 T, considerado de alta resolução naquele período (Figura 1). ${ }^{13}$

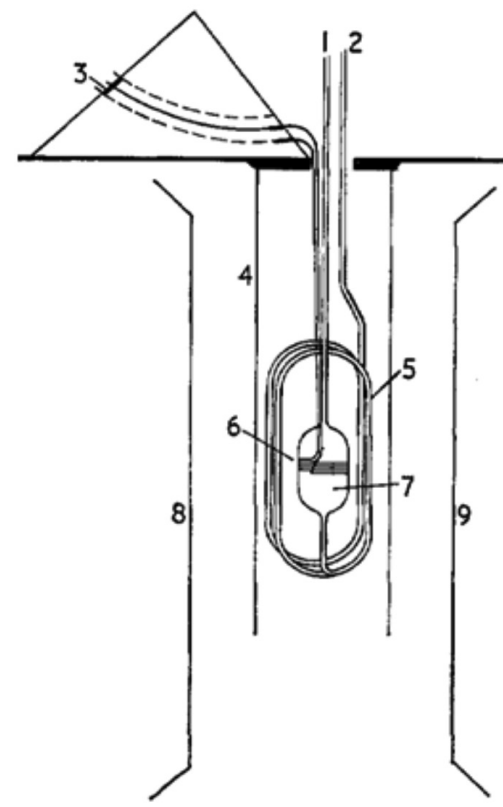

Figura 1. Configuração da sonda de RMN proposta por McIvor, incorporando a região de pré-magnetização: 1 , entrada da amostra em fluxo; 2 , saída da amostra em fluxo; 3, conexão da sonda de RF; 4, proteção metálica; 5 , serpentina de polarização; 6 , bobina de $R F$; 7 , célula de compartimento da amostra; 8 e 9, partes do pólo do magneto. Reproduzida da ref. 13, com permissão da IOPscience Publishing Limited

Nesse trabalho foram realizadas análises em fluxo em alta resolução (largura de linha de 2 a $3 \mathrm{~Hz}$ ) permitindo a quantificação dos componentes de uma mistura de solventes com pequenas diferenças de deslocamento químico, tanto para hidrogênio a $60 \mathrm{MHz}$ quanto flúor, a 59,47 MHz. Para realizar as medidas em fluxo foi necessário que a amostra de solvente passasse por uma serpentina antes de chegar à bobina de detecção do sinal de RMN. A função da serpentina era permitir a circulação da amostra no interior do magneto, de modo a aumentar o tempo de exposição a $\mathrm{B}_{0}$ e garantir que os spins da amostra estivessem totalmente polarizados antes de entrar na bobina de rf (Figura 1). ${ }^{13} \mathrm{O}$ tempo de permanência da amostra no interior do ímã para atingir a condição quantitativa deve ser da ordem de $5 \mathrm{~T}_{1}$, que corresponde à distância/velocidade da amostra antes de chegar à bobina de rf. Essa configuração promoveu um aumento na razão sinal/ ruído e maior precisão nas análises quantitativas e tem sido utilizada em espectrômetros de RMN acoplados a cromatógrafos líquidos de alta eficiência (HPLC). ${ }^{14}$

Em 1971, foi publicado por Sudmeier e Pesek o primeiro estudo de RMN em onda contínua utilizando o método stop and flow, ${ }^{15}$ no qual a amostra em fluxo fica estática no interior da sonda somente durante a aquisição do sinal de RMN. A configuração do sistema consistiu de seringas pneumáticas de $5 \mathrm{~mL}$ montadas em um invólucro de alumínio, permitindo a adição e a retirada de soluções, sem retirar o tubo de amostra da sonda. Nesse trabalho os autores monitoraram a largura de linha de ${ }^{35} \mathrm{Cl}$ em função do tempo após a mistura de $\mathrm{Hg}$ e soro de albumina bovina com vários agentes quelantes, em um estudo de um modelo importante para a terapia de quelação. ${ }^{15}$

Após esses trabalhos, as aplicações da RMN em onda contínua em amostras em fluxo praticamente se encerraram, devido à popularização dos espectrômetros de RMN pulsado. No entanto, recentemente Hills e Wrigth desenvolveram uma nova versão dos aparelhos de RMN em onda contínua para medidas em fluxo. Nesse trabalho demonstraram que a translação da amostra em fluxo pode ser utilizada para observar os sinais de RMN sem a necessidade do pulso de excitação ou gradiente de campo. ${ }^{8}$ Nesse espectrômetro as amostras ao passarem pelo sensor (bobina) são excitadas por $\mathrm{B}_{1} \mathrm{e}$ geram um sinal de RMN que foi utilizado para medir a quantidade de óleo e o BRIX, (teor de sólido solúvel) que está relacionado com o grau de maturação em frutas. ${ }^{8}$

\section{ANÁLISES EM FLUXO POR RMN PULSADA}

Na RMN pulsada não há necessidade de se variar a frequência de excitação ou o campo magnético externo $\mathrm{B}_{0}$, para atingir a condição de RMN (Equação 1), como no modo de onda contínua. A condição de ressonância para todos os sinais de uma amostra é obtida pela banda de excitação do pulso, que deve ser curto o suficiente para irradiar toda a banda espectral a ser analisada.

A principal vantagem do espectrômetro pulsado em relação ao de onda contínua é a alta razão sinal/ruído que se obtém para um mesmo tempo de medida. Nesses espectrômetros, o sinal é adquirido no domínio do tempo, que pode ser usado diretamente nas medidas em fluxo ou serem convertidas para o domínio da frequência através da transformada de Fourier. Quando o sinal é usado diretamente no domínio do tempo (DT) será chamado de RMN-DT e quando se aplica a transformada de Fourier (TF) será denominado de RMN-TF. Como a RMN-DT usa campo magnético bem mais baixo $(<60 \mathrm{MHz})$ e menos homogêneo (largura de linha $>100 \mathrm{~Hz}$ ) do que na RMN-TF, também tem sido chamada de RMN em baixo campo ou RMN em baixa resolução. ${ }^{1}$ Por outro lado, a RMN-TF também tem sido chamada de RMN em alto campo (> $200 \mathrm{MHz}$ ) ou RMN em alta resolução (largura de linha $<1$ $\mathrm{Hz}$ ). Além da espectroscopia, a tomografia de RMN pulsada (TORMN) também tem sido empregada para análises online. ${ }^{16,17}$

A primeira aplicação da RMN pulsada em fluxo foi apresentada por Singer, em 1959, quando estudou o fluxo sanguíneo em cauda de ratos. ${ }^{18} \mathrm{Um}$ dos procedimentos utilizados foi medir o tempo de relaxação longitudinal $\left(\mathrm{T}_{1}\right)$ dos ${ }^{1} \mathrm{H}$ da água no sangue e a mudança desse tempo em função da interrupção do fluxo sanguíneo com um torniquete. Uma vez que o fluxo resulta em menor saturação do sinal da água, ele observou um aumento da amplitude do sinal com o fluxo sanguíneo. ${ }^{18}$

Grover e Singer desenvolveram em 1971 as primeiras aplicações de RMN em fluxo em medicina, realizando medidas da velocidade do fluxo sanguíneo no interior do dedo humano, com a sequência de pulsos eco de spin. ${ }^{19} \mathrm{~A}$ sequência de eco de spin consiste da aplicação de um pulso de $90^{\circ}$ seguido de um pulso de $180^{\circ}$, separados por um intervalo de tempo $\tau$. Essa sequência gera um sinal (eco de spin) após um tempo $\tau$, depois do pulso de $180^{\circ}$, cuja intensidade é proporcional ao tempo de relaxação transversal $\left(\mathrm{T}_{2}\right)$ para as amostras estáticas. No entanto, a amplitude do eco também é dependente do fluxo e, com isso, é possível determinar a distribuição da velocidade de um líquido em movimento. ${ }^{19}$

Em 1978, Watanabe e Niki desenvolveram o primeiro trabalho acoplando RMN-TF com a técnica de HPLC, utilizando um espectrômetro pulsado de $60 \mathrm{MHz}$ para o ${ }^{1} \mathrm{H}$ e o método stop and flow. ${ }^{20}$ Posteriormente, com o aumento da sensibilidade da RMN utilizando 
magnetos supercondutores de alto campo, muitos autores propuseram a realização das análises com os eluentes cromatográficos em fluxo contínuo ${ }^{20}$ Foram desenvolvidos dispositivos especiais para a interface da RMN com a cromatografia para possibilitar a polarização da amostra (Figura 2). ${ }^{21}$ Atualmente, sistemas de HPLC-RMN estão se tornando comuns em laboratórios de pesquisa para isolamento, detecção, quantificação e/ou identificação de compostos, principalmente em análises de produtos naturais, purificação de drogas e metabólitos em fluidos biológicos. ${ }^{14,22-24}$

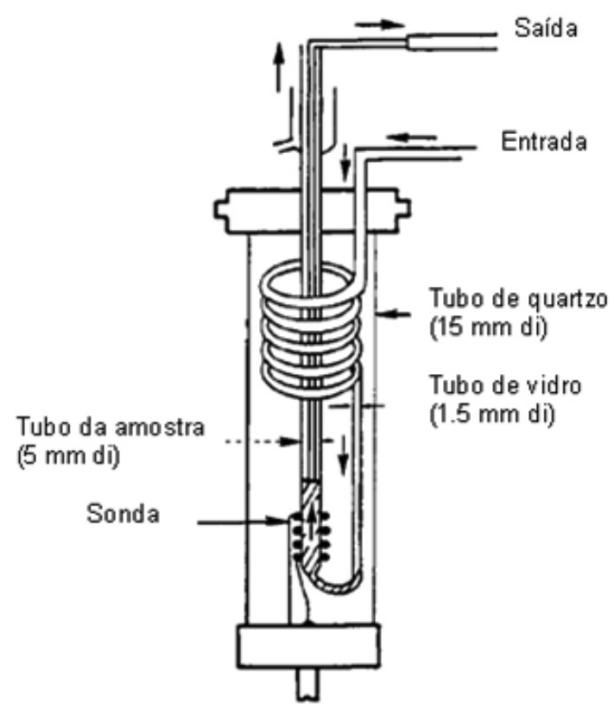

Figura 2. Sistema para acoplamento de RMN-TF em fluxo com o aparelho de $H P L C$, incorporando a região de pré-magnetização. A amostra entra por um tubo espiral de quartzo de $15 \mathrm{~mm}$, que faz com que a amostra se mantenha por mais tempo no interior no magneto, possibilitando sua polarização antes de entrar na região de detecção da sonda. Adaptada da ref. 21, com permissão da American Chemical Society

Pearson et al. descreveram um método pulsado para análise de umidade em trigo. ${ }^{25}$ Seus sensores de RMN, inicialmente construídos para medidas online de umidade em óxido de alumínio, foram adaptados com um dispositivo apropriado para amostra de farinha. Este método era rápido e insensível à densidade de empacotamento da amostra, mas somente apropriado para baixo conteúdo de umidade, devido a não homogeneidade do campo magnético usado. Para eliminar esse efeito, os pesquisadores utilizaram a sequência CPMG (Carr-Purcell-Meiboom-Gill), que é um aperfeiçoamento da sequência de eco de spin. ${ }^{25} \mathrm{Na}$ sequência CPMG após um pulso de excitação de $90^{\circ}$, seguido de um tempo $\tau$, é aplicado um trem de pulsos de refocalização de $180^{\circ}$, defasados em $90^{\circ}$ em relação ao primeiro pulso, separados por um tempo $2 \tau$. Com essa técnica é possível medir todo o decaimento do sinal de RMN em função de $\mathrm{T}_{2}$, mesmo em ímãs de baixa homogeneidade. Atualmente a sequência CPMG é o método clássico de medida de $\mathrm{T}_{2}$ e tem sido empregada para medidas qualitativas e quantitativas de amostras estáticas ou em fluxo, usando espectrômetros de RMN-DT.9,26

No mesmo ano, Renou et al. demonstraram que técnicas pulsadas de RMN-DT em baixo campo permitiram determinações simultâneas de etanol e açúcar em uma fermentação alcoólica média em um tempo curto de análise (3,5 min). ${ }^{27}$

Nicholls e De Los Santos desenvolveram, em 1991, um sensor RMN para medida de umidade para indústria de moagem úmida de milho. ${ }^{28}$ Para isso utilizaram um espectrômetro de $11 \mathrm{MHz}{ }^{1} \mathrm{H}$. Nesse sistema o milho úmido era transportado por uma calha até o sensor de RMN, que era preenchido com a amostra, com a ajuda de um pistão. Após a medida, o pistão retornava a amostra à linha de processo. Os autores utilizaram medidas dos tempos de relaxação $\mathrm{T}_{1}$ e $\mathrm{T}_{2}$ para correlacionar com a umidade da amostra. ${ }^{28}$

Chen et al., em 1993, desenvolveram um sensor de RMN online para avaliação da maturidade de abacates intactos, ${ }^{16}$ utilizando as espectroscopias de RMN-TF, RMN-TD e de TORMN nas análises online. Observaram que havia uma correlação entre a intensidade das imagens TORMN, da razão da área dos sinais de ressonância do óleo em -3,7 ppm e da água em 0 ppm nos espectros de RMN-TF e dos tempos de relaxação $\mathrm{T}_{1}$ e $\mathrm{T}_{2}$ da água na RMN-DT, com o grau de maturação de abacates. Todos os dados foram adquiridos em um espectrômetro de $85 \mathrm{MHz}$ para o ${ }^{1} \mathrm{H}^{16}$

Esse mesmo aparelho foi usado por Zion et al., em 1997, com um sensor de TORMN online para análises qualitativas de azeitonas, ${ }^{17}$ utilizando uma bobina de superfície de $20 \mathrm{~mm}$ de diâmetro e uma bobina de imagem de $150 \mathrm{~mm}$ de diâmetro acoplado. Foram adquiridas projeções unidimensionais do perfil de azeitonas se movendo com velocidades de até $25 \mathrm{~cm} \mathrm{~s}^{-1}$. Nestas análises puderam distinguir azeitonas com e sem caroço. Os erros de classificação para esse experimento foram 4,$3 ; 4,7 ; 2,3$ e $4,0 \%$ para as velocidades da esteira de 0,$5 ; 15$ e $25 \mathrm{~cm} \mathrm{~s}^{-1}$, respectivamente. ${ }^{17}$

Outros pesquisadores também empregaram a RMN online para avaliar qualidade de frutas, medindo o grau de amadurecimento e as injúrias mecânicas e fisiológicas, causadas por microrganismos, entre outros fatores..$^{29-31}$

Um desses estudos, realizado por Hernandez-Sanchez et al., avaliou o escurecimento interno de peras utilizando RMN-DT e TORMN online. As imagens das peras foram adquiridas em velocidade de 54 $\mathrm{mm} \mathrm{s}^{-1}$ e possibilitaram a discriminação entre peras normais e com amolecimento interno. ${ }^{31}$

Prestes et al. também utilizaram a sequência CPMG para medida da qualidade de óleo em sementes intactas no modo stop and flow, empregando um ímã supercondutor de $2,1 \mathrm{~T}$ e $30 \mathrm{~cm}$ de bore. ${ }^{6}$ Demonstraram que o decaimento exponencial $\left(\mathrm{T}_{2}\right)$, obtido com a sequência CPMG, pode ser usado para medir propriedades qualitativas do óleo vegetal para uso em biodiesel como a viscosidade, o índice de iodo e o número de cetano do óleo, diretamente em mais de 1000 sementes por hora. ${ }^{6}$

Andrade et al., em 2011, demonstraram a utilização de uma sequência CPMG com ângulos de refocalização menores que $180^{\circ}$ para reduzir os problemas de sobrecarga do espectrômetro de RMN quando se usa essa sequência para análise em fluxo. ${ }^{26}$ Demonstraram que é possível reduzir a potência de rf em experimentos de CPMG online em cerca de 4 vezes quando se utilizam pulsos de refocalização de $90^{\circ}$. Esse novo método foi denominado de $\mathrm{CPMG}_{90}{ }^{26}$

Em 2011, Osán et al. propuseram um método rápido para medir a velocidade média do fluxo em condições de regime turbulento mesmo sob mudanças rápidas de velocidade de fluxo, usando a sequência de CPMG com velocidade entre 0,8 e 1,65 m s$~^{-1}$, em um espectrômetro de 0,255 T. ${ }^{32}$ Esse método e equipamento também vem sendo utilizado para medida online de petróleo e pode medir o teor de óleo, água e gás de maneira bidirecional. ${ }^{33}$

Além das técnicas convencionais de RMN-DT como a CPMG, também estão sendo desenvolvidas novas sequências de pulsos para medidas online. Dentre elas destaca-se a sequência de Precessão Livre em Onda Contínua, CWFP (Continuous Wave Free Precession). Essa técnica é uma condição especial da técnica RMN no estado estacionário de precessão livre, SSFP (Steady State Free Precession).$^{34}$

\section{CWFP ONLINE}

A sequência CWFP consiste de um trem de pulsos de mesma fase e intensidade, separados por um tempo $\tau<\mathrm{T}_{2}{ }^{*}<<\mathrm{T}_{1}$ e $\mathrm{T}_{2}$, onde $\mathrm{T}_{2}{ }^{*}$ 
é constante de tempo de decaimento livre da indução (FID). Nesta condição, um sinal contínuo é obtido, que não decai com o tempo e cuja amplitude não depende de $T_{1}$, mas da razão $T_{1} / T_{2}$, de acordo com a Equação 2, quando são utilizados pulsos de $90^{\circ}$, onde $\mathrm{M}_{\mathrm{EE}}$ é a intensidade do sinal no estado estacionário e $\mathrm{M}_{0}$ a intensidade do sinal no equilíbrio térmico. ${ }^{35}$

$$
M_{E E}=\frac{M_{0} T_{2}}{\left(T_{1}+T_{2}\right)}
$$

O sinal CWFP é uma combinação dos sinais do FID e eco de spin que, dependendo do ângulo de defasagem $(\psi)$, pode apresentar uma interferência construtiva ou destrutiva das componentes entre si. Quando o ângulo de defasagem $\psi$ é múltiplo ímpar de $\pi$ (Equação 3), o sinal obtido é máximo devido a uma interferência construtiva entre FID e eco. ${ }^{35}$

$$
\Psi=(2 n+1) \pi=\omega \tau
$$

Na Equação 3, $n$ é um número inteiro, $\omega$ é a frequência de ressonância em $\mathrm{rad} \mathrm{s}^{-1} \mathrm{e} \tau$ é o tempo entre os pulsos. Quando o ângulo $\psi$ é múltiplo par de $\pi(\psi=2 n \pi)$, ocorre uma interferência destrutiva entre o FID e o eco que resulta em sinal mínimo. ${ }^{35}$

Azeredo et al. verificaram, em 2001, que a amplitude do sinal CWFP é muito sensível ao fluxo. ${ }^{35} \mathrm{Com}$ o escoamento laminar de água em um espectrômetro de RMN de 7,5 MHz ${ }^{1} \mathrm{H}$ demonstraram que a amplitude do sinal CWFP para uma amostra em fluxo depende da velocidade, do sentido do fluxo, do gradiente de campo magnético, dos tempos de relaxação $\mathrm{T}_{1}$ e $\mathrm{T}_{2}$ da amostra e do ângulo de refocalização. ${ }^{35}$ Como esses fatores são constantes para um dado experimento, Colnago et al. utilizaram a técnica de CWFP para medidas quantitativas em fluxo $\left(13 \mathrm{~cm} \mathrm{~s}^{-1}\right)$ do teor de óleo em sementes. ${ }^{36} \mathrm{Na}$ Figura 3 é apresentado o sistema online para medida do teor de óleo de sementes pela técnica CWFP, baseado em um ímã supercondutor de $2,1 \mathrm{~T}$ e diâmetro interno de $30 \mathrm{~cm}$.

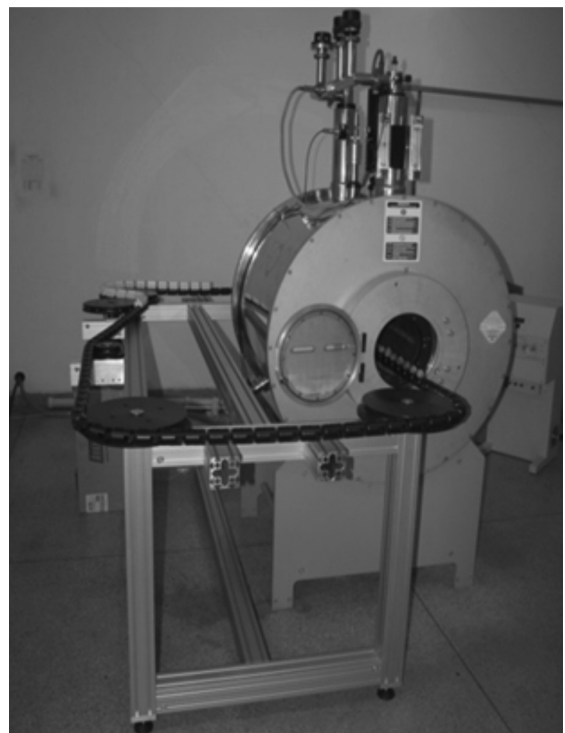

Figura 3. Esteira de poliacetal, usada para medidas online, formada por 105 gomos de $65 \mathrm{~mm}$ atravessando o bore de $30 \mathrm{~cm}$ de diâmetro do magneto de $2,1 \mathrm{~T}$

Na Figura 4 é apresentado o sinal de RMN-CWFP de 99 amostras de sementes obtidas em um tempo total de $14 \mathrm{~s}$. Cada pico representa uma amostra e sua intensidade tem correlação linear com conteúdo de óleo nas sementes, com $\mathrm{r}^{2}=0,95$. Assim, essa técnica tem potencial para determinação do teor de óleo em mais de 20 mil sementes intactas por hora e está sendo utilizada na avaliação do teor de óleo em sementes para seleção de material genético para produção de biodiesel. ${ }^{36}$

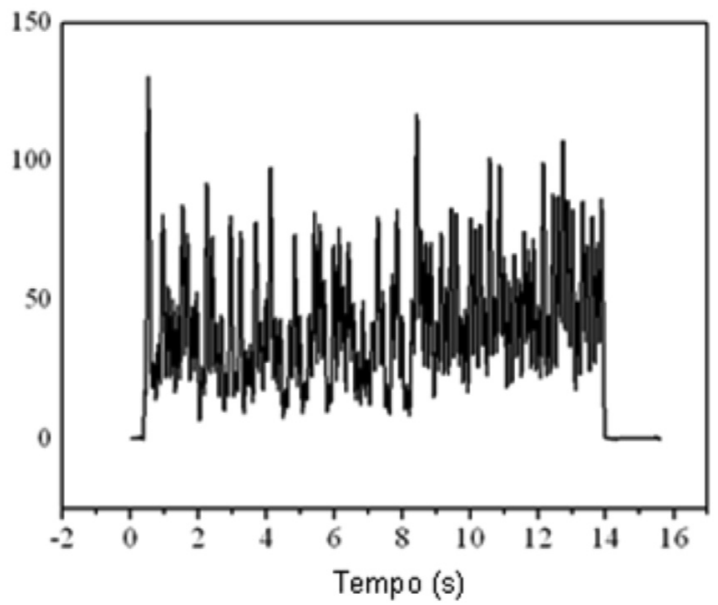

Figura 4. Sinal de RMN online, obtido pela técnica CWPF, de amostras de sementes oleaginosas. Adaptada da ref. 36, com permissão da American Chemical Society

A sequência CWFP também pode ser utilizada para análises qualitativas. ${ }^{37}$ Para isso é necessário utilizar o sinal CWFP desde o primeiro pulso. $\mathrm{O}$ sinal CWFP gerado por um trem de pulsos de $90^{\circ}$ como um tempo $\tau \ll<\mathrm{T}_{2}{ }^{*}$ a partir do equilíbrio térmico passa por dois estados transientes antes de atingir o estado estacionário. O primeiro estado transiente é caracterizado por uma alternância de amplitude do sinal entre valores máximos e mínimos. Este estado é seguido por um estado quasi-estacionário, onde não há alternância na amplitude e o sinal decai exponencialmente com uma constante de tempo $\mathrm{T}^{*}$, que depende de $\mathrm{T}_{1}$ e $\mathrm{T}_{2}$ (Equação 4). Nesta equação, $\alpha$ corresponde ao ângulo do pulso de $\mathrm{B}_{1}$ O estado estacionário CWFP $\left(\mathrm{M}_{\mathrm{EE}}\right)$ é alcançado quando a amplitude do sinal é constante e é dada pela Equação 2.37

$$
T_{(\alpha=\pi / 2)}^{*}=\frac{2 T_{1} T_{2}}{\left(T_{1}+T_{2}\right)}
$$

Combinando as Equações 2 e 4, podem-se determinar os valores de $\mathrm{T}_{1}$ e $\mathrm{T}_{2}$ em um único experimento (Equações 5 e 6 ). ${ }^{37}$

$$
\begin{gathered}
T_{1}=\frac{\frac{T_{(\alpha=\pi / 2)}^{*}}{2}}{\frac{M_{E E}}{M_{0}}} \\
T_{2}=\frac{\frac{T_{(\alpha=\pi / 2)}^{*}}{2}}{1-\frac{M_{E E}}{M_{0}}}
\end{gathered}
$$

e

Assim, Corrêa et al. utilizaram a dependência dos tempos de relaxação $\mathrm{T}_{1}$ e $\mathrm{T}_{2}$ do sinal CWFP para determinação do teor de gordura intramuscular de carne bovina no modo stop and flow. ${ }^{38}$ Demonstraram que o sinal obtido com a sequência CWFP possui maior correlação $(r=0,9)$ com o teor de gordura intramuscular do que o obtido com a sequência CPMG $(r=0,25)$, que depende somente de $\mathrm{T}_{2}{ }^{38}$

Em 2011, Colnago et al. utilizaram a sequência CWFP para medida da qualidade de óleo em sementes com o método stop and flow (Figura 5). ${ }^{39}$ Essas medidas foram realizadas no mesmo sistema online descrito na Figura 3, utilizando 31 amostras de sementes oleaginosas. Na Figura 5 cada pico representa o início do sinal CWFP 
de uma semente, relacionado ao FID e pode ser usado para medir o conteúdo de óleo. Após esse sinal, ocorre o decaimento do estado quasi-estacionário para o estacionário, que depende da razão entre $\mathrm{T}_{1}$ e $\mathrm{T}_{2}$ da semente em questão (Equação 4). No retângulo pontilhado entre 2,95 e 3,35 min estão os sinais do óleo de amostras intactas de macaúba, mamona e melancia, respectivamente; pode-se observar que o sinal do óleo da mamona decaiu mais rápido devido à maior viscosidade do óleo. O óleo da macaúba apresentou uma viscosidade intermediária e a semente de melancia, o óleo menos viscoso com um valor de $\mathrm{T}^{*}$ maior comparado com outras sementes. Os valores de $\mathrm{T}_{2}$ das sementes analisadas com a sequência CWFP apresentaram alta correlação $(r>0,90)$ com os valores medidos pela sequência CPMG, indicando que CWFP pode se utilizado para medir a qualidade de óleo. Esse sistema apresentou um potencial de análise de mais de 1000 amostras por hora. ${ }^{39}$

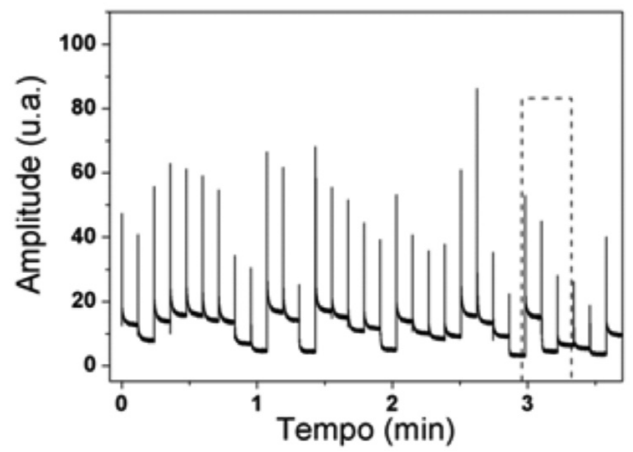

Figura 5. Sinal de 31 amostras de espécies oleaginosas obtidos com a sequência CWFP online utilizando o método stop and flow. Adaptada da ref. 39, com permissão da Wiley

Nesse trabalho também foi utilizado o método de análise de componentes principais, PCA - Principal Component Analysis, para visualização de similaridades ou diferenças entre os sinais CWFP das amostras de sementes analisadas. Os gráficos dos escores de PCA representaram mais de $98 \%$ dos dados $\left(\mathrm{PC}_{1}=95,01 \%\right.$ e $\mathrm{PC}_{2}$ $=3,70 \%$ ) (Figura 6). ${ }^{39}$ As sementes de Fevillea trilobata e café $(\bullet)$, que são compostas por mais de $40 \%$ de ácidos graxos saturados, com razão $\mathrm{T}_{1} / \mathrm{T}_{2}>2$ ficaram do lado esquerdo do gráfico (valores de $\mathrm{PC}_{1}$ de -100 a -80), enquanto que as sementes ricas em ácidos graxos monoinsaturados, com razão $\mathrm{T}_{1} / \mathrm{T}_{2}$ próxima de 2 , isto é, moringa, macadâmia e rabanete $(\boldsymbol{\Lambda})$, ficaram entre -60 a -40 . Entre -20 a +40 ( $\square$ e $\star$ ) ficaram as sementes ricas em ácidos graxos poli-insaturados com razão $T_{1} / T_{2}$ próxima de 1 . A linhaça ( $\star$ ) ficou no topo do lado direito de $\mathrm{PC}_{1}$, devido seu alto conteúdo de ácido linolênico. ${ }^{39}$

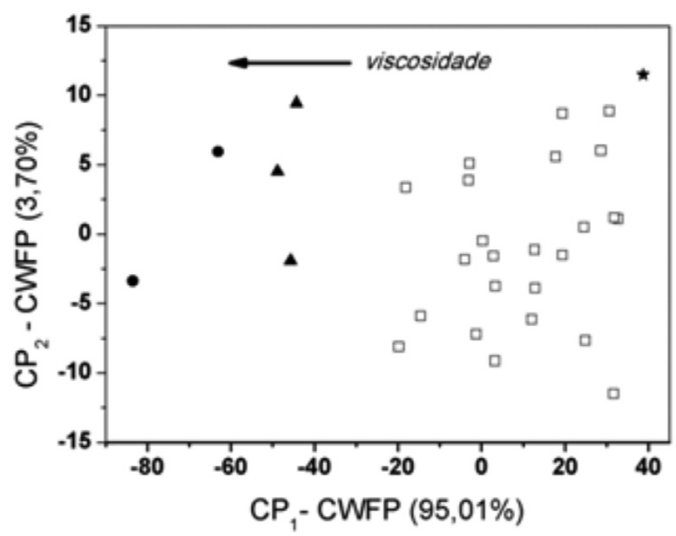

Figura 6. Gráfico dos escores obtidos com a análise por PCA dos dados obtidos por CWFP. Adaptada da ref. 39, com permissão da Wiley
Andrade et al. desenvolveram um novo método para medidas de qualidade de sementes oleaginosas online, baseado na dependência de $\mathrm{T}_{1} \mathrm{e} \mathrm{T}_{2} \cdot{ }^{40}$ Esse método foi denominado CP-CWFP por ser baseado na sequência de eco de spin proposta por Carr-Purcell (CP) e, ainda, por possuir características semelhantes ao método CWFP. A sequência CP-CWFP é mais eficiente do que a CWFP para medida dos tempos de relaxação quando $\mathrm{T}_{1} \sim \mathrm{T}_{2}$. Portanto, esse novo método de medida é mais eficiente para ser usado em espectrômetros de RMN com baixos campos magnéticos $\left(\mathrm{B}_{0}<0,5 \mathrm{~T} \sim 20 \mathrm{MHz}\right.$ para $\left.{ }^{1} \mathrm{H}\right),{ }^{40} \mathrm{uma}$ vez que nessa condição $T_{1}$ e $T_{2}$ tendem a possuir valores similares. Portanto, a sequência CP-CWFP é uma técnica mais robusta para análises em espectrômetros de RMN online com ímãs permanentes, que deverão ser os mais utilizados em controle de qualidade de processos industriais. ${ }^{40}$

Apesar da RMN online em aplicações industriais ainda estar na sua infância, pode-se ver neste artigo que é uma técnica promissora e possui um grande potencial de aplicação no controle e certificação da qualidade de produtos industriais. Assim, espera-se que aparelhos de RMN online comecem a ser mais empregados nos processos industriais principalmente nas indústrias agrícolas, de alimentos e petrolífe$\mathrm{ra}$, onde os produtos naturalmente possuem uma grande variabilidade intrínseca. Portanto, métodos de análise rápidos, automáticos e não destrutivos, como a RMN online deverão ter um grande impacto na produção industrial num futuro próximo.

\section{AGRADECIMENTOS}

À FAPESP, CNPq e FINEP pelo suporte financeiro.

\section{REFERÊNCIAS}

1. Nordon, A.; Mcgill, C. A.; Littlejohn, D.; Analyst 2001, 126, 260.

2. Osiro, D.; Franco, R. W. A.; Colnago, L. A.; J. Braz. Chem. Soc. 2011, $22,1339$.

3. Tavares, L. A.; Ferreira, A. G.; Quim. Nova 2006, 29, 911.

4. Kaiser, C. R.; Quim. Nova 2000, 23, 231.

5. Kotyk, J. J.; Pagel, M. D.; Deppermann, K. L.; Colletti, R. F.; Hoffman, N. G.; Yannakakis, E. J.; Das, P. K.; Ackerman, J. J. K.; J. Am. Oil Chem. Soc. 2005, 82, 885.

6. Prestes, R. A.; Colnago, L. A.; Forato, L. A.; Vizzotto, L.; Novotny, E. H.; Carrilho, E.; Anal. Chim. Acta 2007, 596, 325.

7. Wang, W.; Paliwal, J.; Sensing Instrum. Food Qual. Saf. 2007, 1, 193.

8. Hills, B. P.; Wright, K. M.; J. Magn. Reson. 2006, 178, 193.

9. van Duynhoven, J.; Voda, A.; Witek, M.; van As, H.; Annu. Rep. NMR Spectrosc. 2010, 69, 145.

10. Tellier, C.; Mariette, F.; Annu. Rep. NMR Spectrosc. 1995, 31, 105.

11. Suryan, G.; Proc. Indian Acad. Sci. 1951, 33a, 107.

12. Ladner, W. R.; Stacey, A. E.; Britan. J. Appl. Phys. 1961, 13, 136.

13. Mcivor, M. C. A.; J. Phys. E 1969, 2, 292.

14. Goulas, V.; Gomez-Caravaca, A. M.; Exarchou, V.; Gerothanassis, I. P.; Segura-Carretero, A.; Gutiérrez, A. F.; Food Sci. Technol. 2012, 46, 104.

15. Sudmeier, J. L.; Pesek, J. J.; Inorg. Chem. 1971, 10, 860.

16. Chen, P.; Mccarthy, M. J.; Kauten, R.; Sarig, Y.; Han, S.; J. Agric. Eng. Res. 1993, 55, 177.

17. Zion, B.; Kim, S. M.; Mccarthy, M. J.; Chen, P.; J. Sci. Food Agric. 1997, 75, 496 .

18. Singer, J. R.; Science 1959, 130, 1652.

19. Grover, T.; Singer, J. R.; J. Appl. Phys. 1971, 42, 938.

20. Watanabe, N.; Niki, E.; Proc. Jpn. Acad. 1978, 54b, 194.

21. Haw, J. F.; Glas, T. E.; Hausler, D. W.; Motell, E.; Dorn, H. C.; Anal. Chem. 1980, 52, 1135.

22. Pusecker, K.; Albert, K.; Bayer, E.; J. Chromatogr., A. 1999, 836, 245.

23. Wilson, I. D.; Brinkman, U. A. Th.; Trends Anal. Chem. 2007, $26,847$. 
24. Zhou, G.; Shi, S.; Zhang, W.; Tan, Z.; Chen, Y.; Guo, D.; Zhou, H.; Hu, H.; Tan, J.; Biomed. Chromatogr. 2010, 24, 1130.

25. Pearson, R. M.; Ream, L. R.; Job, C.; Adams, J.; Cereal Foods World 1987, 32, 822.

26. Andrade, F. D.; Netto, A. M.; Colnago, L. A.; Talanta 2011, 84, 84.

27. Renou, J. P.; Briguet, A.; Gatellier, P.; Kopp, J.; Int. J. Food Sci. Technol. 1987, 22, 169.

28. Nicholls, C. I.; De Los Santos, A.; Drying Technol. 1991, 9, 849.

29. Clark, C. J.; Hills, B. P.; Annu. Rep. NMR Spectrosc. 2003, 50, 75.

30. Tu, S. S.; Choi, Y. J.; Mccarthy, M. J.; Mccarthy, K. L.; Postharvest Biol. Technol. 2007, 44, 157.

31. Hernández-Sánchez, N.; Hills, B. P.; Barreiro, P.; Marigheto, N.; Postharvest Biol. Technol. 2007, 44, 260.

32. Osán, T. M.; Ollé, J. M.; Carpinella, M.; Cerioni, L. M. C.; Pusiol, D. J.; Appel, M.; Freeman, J.; Espejo, I.; J. Magn. Reson. 2011, 209, 116.
33. http://nmr-spectrometers.com/, acessada em Agosto 2012.

34. Santos, P. M.; Souza, A. A.; Colnago, L. A.; Quim. Nova 2010, 33, 954.

35. Azeredo, R. B. V.; Engelsberg, M.; Colnago, L. A.; Phys. Rev. E 2001, 64, 16309.

36. Colnago, L. A.; Engelsberg, M.; Souza, A. A.; Barbosa, L. L.; Anal. Chem. 2007, 79, 1271.

37. Venâncio, T.; Engelsberg, M.; Azeredo, R. B. V.; Alem, N. E. R.; Colnago, L.A.; J. Magn. Reson. 2005, 173, 34

38. Corrêa, C. C.; Forato, L. A.; Colnago, L. A.; Anal. Bioanal. Chem. 2009 , 393, 1357.

39. Colnago, L. A.; Azeredo, R. B. V.; Marchi Netto, A.; Andrade, F. D.; Venâncio, T.; Magn. Reson. Chem. 2011, 49, S113.

40. Andrade, F. D.; Marchi Netto, A.; Colnago, L. A.; J. Magn. Reson. 2012, 214,184 